\title{
Characterization of the Maximal Ideal of Operators Associated to the Tensor Norm Defined by an Orlicz Function
}

\begin{abstract}
Given an Orlicz function $H$ satisfying the $\Delta_{2}$ property at zero, one can use the Orlicz sequence space $\ell_{H}$ to define a tensor norm $g_{H}^{c}$ and the minimal ( $H^{c}$-nuclear) and maximal ( $H^{c}$-integral) operator ideals associated to $g_{H}^{c}$ in the sense of Defant and Floret. The aim of this paper is to characterize $H^{c}$-integral operators by a factorization theorem.
\end{abstract}

Keywords: Integral operators, ultraproducts of spaces and maps

AMS subject classification: 46M05, 46A32

\section{Introduction}

The Orlicz theory of function and sequence spaces appears in the literature as a natural attempt to generalize the classical theory of the $L_{p}(\mu)$ and $\ell_{p}$ spaces. In these spaces the role of the function $t^{p}$ is essential and it is quite natural to try to replace this function by a more general one. Moreover, the Orlicz theory has been very fruitful in some basic areas of analysis.

One of the problems in the theory of tensor products and operator ideals in normed spaces is the definition of suitable corresponding norms. In this way, the $\ell_{p}$ spaces play a central role in the definition of interesting topologies in tensor products and operator ideals. In [6, 7] we study the tensor norm with respect to an Orlicz function $H$ and some operator ideals associated to this tensor norm. The so-called "local theory" in Banach spaces, i.e. the study in terms of finite-dimensional subspaces, has so much enriched our understanding of Banach spaces. The ultraproducts technique allows to study some operators in terms of their finite-dimensional parts. In the factorization theorem of $p$-integral operators, which is the key in the proof of many metric properties of the involved tensor norms and operator ideals, the lattice isomorphism between an ultraproduct of $\ell_{p}$ spaces and some $L_{p}(\mu)$ spaces is essential. The structure of the Orlicz sequence spaces is not as simple as that of $\ell_{p}$ spaces; for instance, in general an

G. Loaiza: Univ. EAFIT, Dep. de Ciencias Básicas, Carrera 49 n-7 sur-50 Medellín, Colombia; gloaiza@eafit.edu.co

J. A. López Molina and M. J. Rivera: Univ. Politécnica de Valencia, E.T.S. Ing. Agrón., Camino de Vera 46072 Valencia, Spain; jalopez@mat.upv.es and mjrivera@mat.upv.es. The research of both authors is partially supported by the DGICYT, project PB97-0333. 
ultraproduct of Orlicz sequence spaces does not have a useful representation. The aim of this paper is to obtain a factorization theorem for integral operators of our setting using only "local theory" techniques.

The notation is standard. All the spaces considered are Banach spaces over the real field, since we shall use results in the theory of Banach lattices.

\section{On the norm $g_{H}^{c}$ associated to an Orlicz function $H$ and $H^{c}$-nuclear operators}

A non-degenerated Orlicz function $H$ is a continuous, non-decreasing and convex function defined in $\mathbb{R}^{+}$such that $H(t)=0$ if and only if $t=0$ and $\lim _{t \rightarrow \infty} H(t)=\infty$. All the Orlicz functions $H$ in this paper are non-degenerated and normalized so that $H(1)=1$. The Orlicz sequence space $\ell_{H}$ is the space of all scalar sequences $a=\left(a_{i}\right)_{i=1}^{\infty}$ such that $\sum_{i=1}^{\infty} H\left(\frac{\left|a_{i}\right|}{c}\right)<\infty$ for some $c>0$. The functional

$$
\Pi_{H}(a)=\inf \left\{c>0 \mid \sum_{i=1}^{\infty} H\left(\frac{\left|a_{i}\right|}{c}\right) \leq 1\right\}
$$

is a norm in $\ell_{H}$ and $\left(\ell_{H}, \Pi_{H}(\cdot)\right)$ is a Banach space.

An Orlicz function $H$ satisfies the $\Delta_{2}$ property at zero if the ratio $H(2 t) / H(t)$ is bounded in a neighborhood of $t=0$. Many properties of $\ell_{H}$ show the importance of the behavior of $H$ in a zero neighborhood. For example, generally, the sequence of unit vectors $\left(e_{n}\right)_{n=1}^{\infty}$ is not a basis for $\left(\ell_{H}, \Pi_{H}(\cdot)\right)$, and if the closure of the linear span of $\left(e_{n}\right)_{n=1}^{\infty}$ in $\ell_{H}$ is denoted by $h_{H}$, it is known that $\ell_{H}=h_{H}$ if and only if $H$ satisfies the $\Delta_{2}$ property at zero.

In the duality theory of Orlicz spaces, the notion of a function complementary to an Orlicz function $H$ is essential. An Orlicz function $H^{*}$ is said to be the complementary of $H$ if

$$
H^{*}(u)=\max \{u t-H(t): 0<t<\infty\} .
$$

Moreover, $H^{* *}=H$. With the aid of $H^{*}$ we can introduce another equivalent norm in $\ell_{H}$ defined as

$$
\|a\|_{H}=\sup \left\{\sum_{n=1}^{\infty} a_{n} b_{n} \mid \Pi_{H}\left(\left(b_{n}\right)\right) \leq 1\right\}
$$

if $a=\left(a_{n}\right)_{n=1}^{\infty}$, having the property that $\left(h_{H}, \Pi_{H}(\cdot)\right)^{\prime}=\left(\ell_{H^{*}},\|\cdot\|_{H^{*}}\right)$ as isometric spaces. Moreover, $\ell_{H}$ is reflexive if and only if both $H$ and $H^{*}$ satisfy the $\Delta_{2}$-property at zero. For more information on Orlicz functions and Orlicz sequence spaces the reader is referred to [5].

Given a Banach space $E$, a sequence $\left(x_{n}\right)_{n=1}^{\infty} \subset E$ is

- strongly $H$-summing if $\pi\left(\left(x_{n}\right)\right)=\Pi_{H}\left(\left(\left\|x_{n}\right\|\right)\right)<\infty$

- weakly $H$-summing if $\varepsilon\left(\left(x_{n}\right)\right)=\sup _{\left\|x^{\prime}\right\| \leq 1}\left\|\left(\left|\left\langle x_{n}, x^{\prime}\right\rangle\right|\right)\right\|_{H}<\infty$.

If $\Gamma$ is a set, $\ell_{H}(\Gamma)$ denotes the set of elements $a=\left(a_{\gamma}\right)_{\gamma \in \Gamma}$ with $a_{\gamma} \in \mathbb{R}$ for every $\gamma \in \Gamma$, so that there is a sequence $S=\left\{\gamma_{n}\right\}_{n \geq 1}$ in $\Gamma$ with $a_{\gamma}=0$ if $\gamma \notin S$, endowed with the 
norm $\Pi_{H}(a)=\Pi_{H}\left(\left(a_{\gamma_{n}}\right)\right)$. If $\left\{X_{\gamma}\right\}_{\gamma \in \Gamma}$ is a family of Banach spaces, $\ell_{H}\left\{\left(\left(X_{\gamma}\right)_{\gamma \in \Gamma}\right)\right\}$ denotes the Bochner space of elements $x=\left(x_{\gamma}\right)_{\gamma \in \Gamma}$ with $x_{\gamma} \in X_{\gamma}$ for every $\gamma \in \Gamma$, such that $\left(\left\|x_{\gamma}\right\|\right)_{\gamma \in \Gamma} \in \ell_{H}(\Gamma)$, with the norm $\pi_{H}(x)=\Pi_{H}\left(\left(\left\|x_{\gamma}\right\|\right)_{\gamma \in \Gamma}\right)$. If $X_{\gamma}=X$ for each $\gamma \in \Gamma$, we write $\ell_{H}\{\Gamma, X\}$ instead of $\ell_{H}\left\{\left(\left(X_{\gamma}\right)_{\gamma \in \Gamma}\right)\right\}$. If $\Gamma=\mathbb{N}$, then $\ell_{H}\left\{\left(X_{n}\right)\right\}$ and $\ell_{H}\{X\}$ are written instead of $\ell_{H}\left\{\left(X_{n}\right)_{n \in \mathbb{N}}\right\}$ and $\ell_{H}\{\mathbb{N}, X\}$, respectively.

The definitions and results in the theory of tensor norms and operator ideals involved in this paper are exposed in [1]. Given a pair of Banach spaces $E$ and $F$ and a tensor norm $\alpha, E \otimes_{\alpha} F$ represents the space $E \otimes F$ endowed with the $\alpha$-normed topology. The completion of $E \otimes_{\alpha} F$ is denoted by $E \hat{\otimes}_{\alpha} F$, and the norm of $z$ in $E \hat{\otimes}_{\alpha} F$ by $\alpha(z ; E \otimes F)$. If there is no risk of mistake we write $\alpha(z)$ instead of $\alpha(z ; E \otimes F)$. This is recalled from the metric mapping property: if $A_{i} \in \mathcal{L}\left(E_{i 1}, E_{i 2}\right)(i=1,2)$, then $A_{1} \otimes A_{2} \in \mathcal{L}\left(E_{11} \otimes_{\alpha} E_{21}, E_{12} \otimes_{\alpha} E_{22}\right)$ with $\left\|A_{1} \otimes A_{2}\right\| \leq\left\|A_{1}\right\|\left\|A_{2}\right\|$.

Definition 1. Let $E$ and $F$ be Banach spaces. For every $z \in E \otimes F$ we define

$$
g_{H}(z)=\inf \left\{\pi_{H}\left(\left(x_{n}\right)\right) \varepsilon_{H^{*}}\left(\left(y_{n}\right)\right) \mid z=\sum_{n=1}^{m} x_{n} \otimes y_{n}\right\} .
$$

It is possible that for some Orlicz function $H$ the functional $g_{H}$ does not satisfy the triangular inequality, but it is always a quasi-norm for $E \otimes F$ to posses the metric mapping property. In order to get a tensor norm it is necessary to do the convexification $g_{H}^{c}$ of $g_{H}$, so that

$$
g_{H}^{c}(z)=\inf \left\{\sum_{i=1}^{n} \pi_{H}\left(\left(x_{i j}\right)\right) \varepsilon_{H^{*}}\left(\left(y_{i j}\right)\right) \mid z=\sum_{i=1}^{n} \sum_{j=1}^{m} x_{i j} \otimes y_{i j}\right\} .
$$

A suitable representation of the elements of a completed tensor product is a basic tool in the study of the involved operator ideals. The reader is referred to [6], to prove that if a Orlicz function $H$ satisfies the $\Delta_{2}$ property at zero and $z \in E \hat{\otimes}_{g_{H}^{c}} F$, there are $\left\{\left(x_{i j}\right)_{j=1}^{\infty}\right\}_{i \in \mathbb{N}} \subset E^{\mathbb{N}}$ and $\left\{\left(y_{i j}\right)_{j=1}^{\infty}\right\}_{i \in \mathbb{N}} \subset F^{\mathbb{N}}$ such that

$$
\sum_{i=1}^{\infty} \pi_{H}\left(\left(x_{i j}\right)\right) \varepsilon_{H^{*}}\left(\left(y_{i j}\right)\right)<\infty \quad \text { and } \quad z=\sum_{i, j=1}^{\infty} x_{i j} \otimes y_{i j} .
$$

Moreover,

$$
g_{H}^{c}(z)=\inf \sum_{i=1}^{\infty} \pi_{H}\left(\left(x_{i j}\right)\right) \varepsilon_{H^{*}}\left(\left(y_{i j}\right)\right)
$$

where the infimum is taken over all such representations of $z$.

From now on the Orlicz function $H$ satisfies the $\Delta_{2}$ property at zero.

Every representation of $z \in E^{\prime} \hat{\otimes}_{g_{H^{c}}} F$,

$$
z=\sum_{i, j=1}^{\infty} x_{i j}^{\prime} \otimes y_{i j} \quad \text { with } \quad \sum_{i=1}^{\infty} \pi_{H}\left(\left(x_{i j}^{\prime}\right)\right) \varepsilon_{H^{*}}\left(\left(y_{i j}\right)\right)<\infty
$$


defines an operator $T_{z} \in \mathcal{L}(E, F)$ such that

$$
T_{z}(x)=\sum_{i, j=1}^{\infty}\left\langle x_{i j}^{\prime}, x\right\rangle y_{i j} \quad(x \in E)
$$

We remark that all possible representations of $z$ define the same map $T_{z}$. Let $\Phi_{E F}$ : $E^{\prime} \hat{\otimes}_{g_{H}^{c}} F \rightarrow \mathcal{L}(E, F)$ with $\Phi_{E F}(z)=T_{z}$.

Definition 2. Let $E$ and $F$ be Banach spaces. An operator $T: E \rightarrow F$ is said to be $H^{c}$-nuclear if $T=\Phi_{E F}(z)$ for some $z \in E^{\prime} \hat{\otimes}_{g_{H}^{c}} F$.

$\mathcal{N}_{H^{c}}(E, F)$ denotes the space of $H^{c}$-nuclear operators $T: E \rightarrow F$ endowed with the topology of the norm

$$
\mathbf{N}_{H^{c}}(T)=\inf \left\{\sum_{i=1}^{\infty} \pi_{H}\left(\left(x_{i j}^{\prime}\right)\right) \varepsilon_{H^{*}}\left(\left(y_{i j}\right)\right) \mid \Phi_{E F}(z)=T, z=\sum_{i, j=1}^{\infty} x_{i j}^{\prime} \otimes y_{i j}\right\} .
$$

For every pair of Banach spaces $E$ and $F,\left(\mathcal{N}_{H^{c}}(E, F), \mathbf{N}_{H^{c}}\right)$ is a component of the minimal operator ideal $\left(\mathcal{N}_{H^{c}}, \mathbf{N}_{H^{c}}\right)$ associated to $g_{H}^{c}$, called the ideal of $H^{c}$-nuclear operators.

The following characterization of $H^{c}$-nuclear operators is proved in [7]:

Theorem 3. For every pair of Banach spaces $E$ and $F$, let $T$ be an operator in $\mathcal{L}(E, F)$. Then the following assertions are equivalent:

1) $T$ is $H^{c}$-nuclear.

2) $T$ factors in the following way:

where $B$ is a diagonal multiplication operator defined by a positive sequence $\left(\left(b_{i j}\right)\right) \in$ $\ell_{1}\left\{\ell_{H}\right\}$.

Furthermore, $\mathbf{N}_{H^{c}}(T)=\inf \{\|D\|\|B\|\|A\|\}$, taking the infimum over all such factors. 


\section{3. $H^{c}$-integral operators}

According to the general theory of tensor norms and operator ideals, the ideal $\left(\mathcal{I}_{H^{c}}, \mathbb{I}_{H^{c}}\right)$ of $H^{c}$-integral operators is the maximal operator ideal associated to that of $H^{c}$-nuclear operators. According to [1], for every pair of Banach spaces $E$ and $F$, an operator $T: E \rightarrow F$ is $H^{c}$-integral if and only if $J_{F} T \in\left(E \otimes_{\left(g_{H}^{c}\right)^{\prime}} F^{\prime}\right)^{\prime}$, where $J_{F}: F \rightarrow F^{\prime \prime}$ is the canonical isometric map. The aim of this paper is to obtain the characterization of $H^{c}$-integral operators by means of a factorization theorem.

Theorem 4. Let $G$ be an abstract $M$-space. Then every positive operator $T: G \rightarrow$ $\ell_{1}\left\{\ell_{H}\left(\Gamma_{i}\right)\right\}$ is $H^{c}$-integral.

Proof. As $\ell_{1}\left\{\ell_{H}\left(\Gamma_{i}\right)\right\}=\left(c_{0}\left\{h_{H^{*}}\left(\Gamma_{i}\right)\right\}\right)^{\prime}$, then $\ell_{1}\left\{\ell_{H}\left(\Gamma_{i}\right)\right\}$ is complemented in its bidual space $\left(\ell_{1}\left\{\ell_{H}\left(\Gamma_{i}\right)\right\}\right)^{\prime \prime}$ with a positive projection $P:\left(\ell_{1}\left\{\ell_{H}\left(\Gamma_{i}\right)\right\}\right)^{\prime \prime} \rightarrow \ell_{1}\left\{\ell_{H}\left(\Gamma_{i}\right)\right\}$. In consequence, the map $P T^{\prime \prime}: G^{\prime \prime} \rightarrow \ell_{1}\left\{\ell_{H}\left(\Gamma_{i}\right)\right\}$ is positive. As $G$ is an abstract $M$ space, then $G^{\prime}$ is lattice isomorphic to $L_{1}(\mu)$ for some measure space $(\Omega, \Sigma, \mu)$ (see [9: Theorem 8.5]), hence $G^{\prime \prime}$ is lattice isomorphic to $L_{\infty}(\mu)$. Let $B: G^{\prime \prime} \rightarrow L_{\infty}(\mu)$ denote the corresponding positive isometric map and $I_{G}: G \rightarrow G^{\prime \prime}$ the canonical inclusion map. But $T=P T^{\prime \prime} B^{-1} B I_{G}$ with $P T^{\prime \prime} B^{-1}: L_{\infty}(\mu) \rightarrow \ell_{1}\left\{\ell_{H}\left(\Gamma_{i}\right)\right\}$, hence we only have to see that every positive map $S: L_{\infty}(\mu) \rightarrow \ell_{1}\left\{\ell_{H}\left(\Gamma_{i}\right)\right\}$ is $H^{c}$-integral. But as the unit vectors system $\left\{e_{i g}\right\}_{g \in \Gamma_{i}(i \in \mathbb{N})}$ is a basis for $\ell_{1}\left\{\ell_{H}\left(\Gamma_{i}\right)\right\}$, if $S\left(\chi_{\Omega}\right)=\sum_{r, s=1}^{\infty} w_{r s} e_{i(r) g(s)}$, then $\left\{e_{i(r) g(s)}\right\}_{r, s \in \mathbb{N}}$ is a basis for the space image and so it is enough to see that every positive operator $S: L_{\infty}(\mu) \rightarrow \ell_{1}\left\{\ell_{H}\right\}$ is $H^{c}$-integral.

Let $\mathcal{T}$ denote the linear span of $\left\{e_{i j}\right\}_{i, j \in \mathbb{N}}$, which is dense in $c_{0}\left\{h_{H^{*}}\right\}$. From the density lemma we only have to see that $S \in\left(L_{\infty}(\mu) \otimes_{\left(g_{H}^{c}\right)^{\prime}} \mathcal{T}\right)^{\prime}$. Given an arbitrary element $z \in L_{\infty}(\mu) \otimes_{\left(g_{H}^{c}\right)^{\prime}} \mathcal{T}$, let $X$ and $Y$ be finite-dimensional subspaces of $L_{\infty}(\mu)$ and $\mathcal{T}$, respectively. If the system $\left\{g_{s}\right\}_{s=1}^{m}$ is a basis for $Y$ such that $g_{s}=$ $\sum_{u=1}^{k} \sum_{v=l}^{r} c_{s u v} e_{i(s u) j(s v)}$, every $g \in Y$ can be expressed as $g=\sum_{h=1}^{n} \sum_{w=1}^{t} b_{h w} e_{i_{h} j_{w}}$. Once $g \in Y$ and $f \in X$ have been fixed, we have

$$
\begin{aligned}
\langle S, f \otimes g\rangle & =\langle S(f), g\rangle \\
& =\left\langle f, S^{\prime}(g)\right\rangle \\
& =\left\langle f, \sum_{h=1}^{m} \sum_{w=1}^{t} b_{h w} S^{\prime}\left(e_{i_{h} j_{w}}\right)\right\rangle \\
& =\left\langle f, \sum_{h=1}^{n} \sum_{w=1}^{t} b_{h w}\left\langle e_{i_{h} j_{w}}, e_{i_{h} j_{w}}\right\rangle S^{\prime}\left(e_{i_{h} j_{w}}\right)\right\rangle \\
& =\left\langle f \otimes g, \sum_{h=1}^{n} \sum_{w=1}^{t} S^{\prime}\left(e_{i_{h} j_{w}}\right) \otimes e_{i_{h} j_{w}}\right\rangle
\end{aligned}
$$

so that

$$
U=\sum_{h=1}^{n} \sum_{w=1}^{t} S^{\prime}\left(e_{i_{h} j_{w}}\right) \otimes e_{i_{h} j_{w}} \in L_{\infty}(\mu) \otimes \ell_{1}\left\{\ell_{H}\right\}
$$


Then for linearity, $\langle z, S\rangle=\langle U, z\rangle$ for every $z \in X \otimes Y_{i}$. But

$$
\begin{aligned}
g_{H^{c}} & \left(U ; L_{\infty}(\mu) \otimes \ell_{1}\left\{\ell_{H}\right\}\right) \\
& \leq \sum_{h=1}^{n} \pi_{H}\left(\left(S^{\prime}\left(e_{i_{h} j_{w}}\right)\right)\right) \varepsilon_{H^{*}}\left(\left(e_{i_{h} j_{w}}\right)\right) \\
& =\sum_{h=1}^{n} \inf \left\{\rho>0 \mid \sum_{w=1}^{t} H\left(\frac{\left\|S^{\prime}\left(e_{i_{h} j_{w}}\right)\right\|}{\rho}\right) \leq 1\right\} \sup _{\left\|h^{*}\right\|_{H^{*}} \leq 1}\left\|\left(\left\langle h^{*}, e_{\left.i_{h} j_{w}\right)}\right\rangle\right)\right\|_{H^{*}} \\
& \leq \sum_{h=1}^{n} \inf \left\{\rho>0 \mid \sum_{w=1}^{t} H\left(\frac{\left|\left\langle S^{\prime}\left(e_{i_{h} j_{w}}\right), \chi_{\Omega}\right\rangle\right|}{\rho}\right) \leq 1\right\} \\
& =\sum_{h=1}^{n} \inf \left\{\rho>0 \mid \sum_{w=1}^{t} H\left(\frac{\left|\left\langle e_{i_{h} j_{w}}, S\left(\chi_{\Omega}\right)\right\rangle\right|}{\rho}\right) \leq 1\right\} \\
& \leq\left\|S\left(\chi_{\Omega}\right)\right\|_{\ell_{1}\left\{\ell_{H}\right\}} \\
\leq & \|S\|
\end{aligned}
$$

and then $S$ is $H^{c}$-integral, with an integral norm smaller than or equal to $\|S\|$

\section{Definition 5.}

a) A Banach space $X$ is said to be finitely representable in a family of Banach spaces $\left\{X_{i}\right\}_{i \in I}$ if, for every finite-dimensional subspace $M$ of $X$ and for every $\varepsilon>0$, there are an index $i \in I$ and a finite-dimensional subspace $N$ of $X_{i}$ such that the Banach-Mazur distance $d(M, N) \leq 1+\varepsilon$.

b) A Banach lattice $X$ is said to be lattice finitely representable in a family of Banach lattices $\left\{X_{i}\right\}_{i \in I}$ if, for every finite-dimensional sublattice $M$ of $X$ and for every $\varepsilon>0$, there are an index $i \in I$, a finite-dimensional sublattice $N$ of $X_{i}$ and a lattice isomorphism $J: M \rightarrow N$ so that $\|J\|\left\|J^{-1}\right\| \leq 1+\varepsilon$.

If for every $i \in I$ the space $X_{i}$ is a subspace (sublattice) of a Banach space (lattice) $Y$, then $X$ is said to be (lattice) finitely representable in $Y$.

The main result achieved in this paper is as follows.

Theorem 6. Let $G$ and $X$ be Banach lattices such that $G$ is an abstract $M$-space and $X$ is lattice finitely representable in $\ell_{1}\left\{\ell_{H}\right\}$. Then every lattice homomorphism $T: G \rightarrow X$ is $H^{c}$-integral.

To obtain this theorem, we must first consider the result given in [2: Lemma 4.4]:

Lemma 7. Let $G$ be an order complete Banach lattice and let $X$ be a finitedimensional Banach subspace of $G$. Then for every $\varepsilon>0$ there is a finite-dimensional Banach sublattice $Y$ of $G$ and an operator $A: X \rightarrow Y$ such that $\|A(x)-x\| \leq \varepsilon\|x\|$ for all $x \in X$.

Remark 8. The inequalities $\|A\| \leq 1+\varepsilon$ and $\left\|A-\operatorname{id}_{X}\right\| \leq \varepsilon$ are easily demonstrated.

Definition 9. A Banach space $E$ is said to be a $\mathcal{L}_{p, \lambda}$-space $(1 \leq p \leq \infty$ and $1 \leq \lambda<\infty)$, if for every finite-dimensional subspace $P$ of $E$ and for every $\varepsilon>0$ there is 
a finite-dimensional subspace $Q$ of $E$ containing $P$ such that the Banach-Mazur distance $d\left(Q, \ell_{p}^{\operatorname{dim}(Q)}\right)<\lambda$

It is known that $L_{p}(\mu)$ are $\mathcal{L}_{p, \lambda}$-spaces for every $\lambda>1$. The next proposition involving $\mathcal{L}_{\infty, \lambda}$ spaces is an extension of a well known result of Hollstein [4: Proposition $2.2]$. For every Banach space $F$ and for every closed subspace $F_{0}$ of $F, K_{F_{0}}: F \rightarrow F / F_{0}$ represents the canonical quotient map, and the open unit ball in $F$ is denoted by $B_{F}$.

Proposition 10. Let $E$ be a $\mathcal{L}_{\infty, \lambda}$-space. Then, for every finitely generated tensor norm $\alpha, E \otimes_{\alpha} \cdot$ isomorphically respects quotients. More precisely, for every Banach space $F$ and for every closed subspace $F_{0}$ of $F$,

$$
\stackrel{\circ}{B}_{E \otimes_{\alpha} F / F_{0}} \subset \lambda\left(\mathrm{id}_{E} \otimes K_{F_{0}}\right)\left(\stackrel{\circ}{B}_{E \otimes_{\alpha} F}\right) .
$$

Proof. We have to see that for every $v \in E \otimes F / F_{0}$ there is $u \in E \otimes F$ so that

$$
\left(i d_{E} \otimes K_{F_{0}}\right)(u)=v \quad \text { and } \quad \alpha(u ; E \otimes F) \leq \lambda \alpha\left(v ; E \otimes F / F_{0}\right) .
$$

Given $v \in E \otimes F / F_{0}$ and $\varepsilon>0$, let

$$
v=\sum_{i=1}^{n} x_{i} \otimes z_{i} \in E \otimes F / F_{0}
$$

a representation of $v \in E \otimes F / F_{0}$ such that the vectors $z_{i}(i=1, \ldots, n)$ are linearly independent in $F / F_{0}$. Let $M$ and $P$ be finite-dimensional subspaces of $E$ and $F / F_{0}$, respectively, so that

$$
\alpha(v ; M \otimes P) \leq \alpha\left(v ; E \otimes F / F_{0}\right)+\varepsilon .
$$

We remark that $p=\operatorname{dim}(P) \geq n$. For each $j=1,2, \ldots, p$ there is $y_{j} \in F$ such that $\left\{K_{F_{0}}\left(y_{j}\right)\right\}_{j=1}^{p}$ is a basis for $P, K_{F_{0}}\left(y_{j}\right)=z_{j}$, and $\left\|y_{j}\right\| \leq\left\|z_{j}\right\|+1$ if $1 \leq j \leq n$. Let $N \subset F$ be the linear subspace of $F$ generated by the linearly independent system $\left\{y_{j}\right\}_{j=1}^{p}$. The map $R=\left(K_{F_{0}}\right)_{\mid N}, R: N \rightarrow P$ is bijective and continuous with a norm value less or equal to one. But also for every $\hat{y} \in P^{\prime}$,

$$
\left\|R^{\prime}(\hat{y})\right\|_{N^{\prime}}=\sup _{\|n\|_{N}<1}\left|\left\langle R^{\prime}(\hat{y}), n\right\rangle\right|=\sup _{\|n\|_{N}<1}\left|\left\langle\hat{y}, K_{F_{0}}(n)\right\rangle\right|=\|\hat{y}\|_{P^{\prime}}
$$

because $K_{F_{0}}$ is a quotient map and then $K_{F_{0}}\left(\stackrel{\circ}{B}_{N}\right)=\stackrel{\circ}{B}_{P}$, hence $R^{\prime}$ and $R$ are isometric maps.

We consider the tensor

$$
u=\sum_{j=1}^{n} x_{j} \otimes y_{j} \in M \otimes N \subset E \otimes F .
$$

It is clear that

$$
\left(I_{E} \otimes R\right)(u)=v \in E \otimes F / F_{0} .
$$

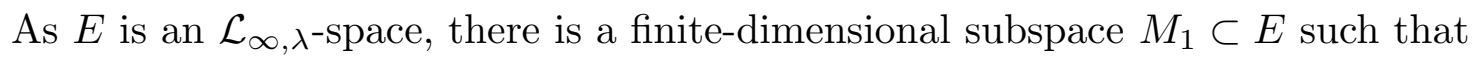

$$
M \subset M_{1}
$$


and an isomorphism $T: M_{1} \rightarrow \ell_{\infty}^{m_{1}}\left(\right.$ where $m_{1}=\operatorname{dim}\left(M_{1}\right)$ ) such that

$$
\|T\|\left\|T^{-1}\right\| \leq \lambda
$$

From Lemma 7, as $\ell_{\infty}^{m_{1}}$ is an order continuous Banach lattice, there is a sublattice $M_{2} \subset \ell_{\infty}^{m_{1}}$ and an operator $A: T(M) \rightarrow M_{2}$ so that

$$
\|A(x)-x\| \leq \varepsilon\|x\| \quad(x \in T(M))
$$

Then we have

$$
\begin{aligned}
& \alpha(u ; E \otimes F) \\
& \quad \leq \alpha\left(u ; M_{1} \otimes N\right) \\
& \quad=\alpha\left(\left(T^{-1} \otimes i d_{N}\right)\left(T \otimes i d_{N}\right)(u) ; M_{1} \otimes N\right) \\
& \quad \leq\left\|T^{-1}\right\| \alpha\left(\left(T \otimes i d_{N}\right)(u): \ell_{\infty}^{m_{1}} \otimes N\right) \\
& \quad \leq\left\|T^{-1}\right\|\left(\alpha\left(\left(\left(i d_{T(M)}-A\right) T \otimes i d_{N}\right)(u) ; \ell_{\infty}^{m_{1}} \otimes N\right)+\alpha\left(\left(A T \otimes i d_{N}\right)(u) ; \ell_{\infty}^{m_{1}} \otimes N\right)\right) \\
& \quad \leq\left\|T^{-1}\right\|\left(\alpha\left(\left(\left(i d_{T(M)}-A\right) T \otimes i d_{N}\right)(u) ; M_{2} \otimes N\right)+\alpha\left(\left(A T \otimes i d_{N}\right)(u) ; M_{2} \otimes N\right)\right) \\
& \quad=\left\|T^{-1}\right\|\left(\alpha\left(\left(\left(i d_{T(M)}-A\right) T \otimes R\right)(u) ; M_{2} \otimes P\right)+\alpha\left((A T \otimes R)(u) ; M_{2} \otimes P\right)\right) \\
& \quad=\left\|T^{-1}\right\|\left(\alpha\left(\left(\left(i d_{T(M)}-A\right) T \otimes i d_{P}\right)(v) ; M_{2} \otimes P\right)+\alpha\left(\left(A T \otimes i d_{P}\right)(v) ; M_{2} \otimes P\right)\right) .
\end{aligned}
$$

As $M_{2}$ is a complemented subspace $\ell_{\infty}^{m_{1}}$ with projection $S: \ell_{\infty}^{m_{1}} \rightarrow M_{2}$ such that $\|S\| \leq 1$ (see [5: p. 162]), using Remark 8 and (3), then

$$
\begin{aligned}
& \alpha(u ; E \otimes F) \\
& \quad \leq\left\|T^{-1}\right\|\left(\alpha\left(\left(\left(i d_{T(M)}-A\right) T \otimes i d_{P}\right)(v) ; M_{2} \otimes P\right)+\alpha\left(\left(A T \otimes i d_{P}\right)(v) ; M_{2} \otimes P\right)\right) \\
& \quad \leq\left\|T^{-1}\right\|\|S\|\left(\alpha\left(\left(\left(i d_{T(M)}-A\right) T \otimes i d_{P}\right)(v) ; \ell_{\infty}^{m_{1}} \otimes P\right)+\alpha\left(\left(A T \otimes i d_{P}\right)(v) ; \ell_{\infty}^{m_{1}} \otimes P\right)\right) \\
& \quad \leq\left\|T^{-1}\right\|\left(\left\|i d_{T(M)}-A\right\|\|T\| \alpha\left(v ; M_{1} \otimes P\right)+\|A\|\|T\| \alpha\left(v ; M_{1} \otimes P\right)\right) \\
& \quad \leq\left\|T^{-1}\right\|\|T\|(1+\varepsilon) \alpha\left(v ; M_{1} \otimes P\right) \\
& \quad \leq \lambda(1+\varepsilon)\left(\alpha\left(v ; E \otimes F / F_{0}\right)+\varepsilon\right) .
\end{aligned}
$$

Hence $\alpha(u ; E \otimes F) \leq \lambda \alpha\left(v ; E \otimes F / F_{0}\right)$

Proof of Theorem 6. As $G$ is an abstract $M$-space, then $G^{\prime \prime}$ is lattice isomorphic to $L_{\infty}(\mu)$ for some measure space $(\Omega, \Sigma, \mu)$. Let $B: G \rightarrow L_{\infty}(\mu)$ denote the corresponding positive isometric map. Hence $J_{X} T=T^{\prime \prime} B$, where $J_{X}: X \rightarrow X^{\prime \prime}$ is the canonical isometric map. Then we only have to see that $T^{\prime \prime} \in\left(L_{\infty}(\mu) \otimes_{\left(g_{H}^{c}\right)^{\prime}} X^{\prime}\right)^{\prime}$.

Given $z \in L_{\infty}(\mu) \otimes X^{\prime}$ and $\varepsilon>0$, let $M \subset L_{\infty}(\mu)$ and $N \subset X^{\prime}$ be finite-dimensional subspaces and let $z=\sum_{i=1}^{n} f_{i} \otimes x_{i}^{\prime}$ be a fixed representation of $z$ with $f_{i} \in M$ and $x_{i}^{\prime} \in N(i=1, \ldots, n)$ such that $\left(g_{H}^{c}\right)^{\prime}(z ; M \otimes N) \leq\left(g_{H}^{c}\right)^{\prime}\left(z ; L_{\infty}(\mu) \otimes X^{\prime}\right)+\varepsilon$. Let $M_{1}$ 
be a finite-dimensional sublattice of $L_{\infty}(\mu)$ and $A: M \rightarrow M_{1}$ an operator so that, for every $f \in M,\|A(f)-f\| \leq \varepsilon\|f\|$. Then

$$
\begin{aligned}
\left|\left\langle T^{\prime \prime}, z\right\rangle\right| & =\left|\sum_{i=1}^{n}\left\langle T^{\prime \prime}\left(f_{i}\right), x_{i}^{\prime}\right\rangle\right| \\
& \leq\left|\sum_{i=1}^{n}\left\langle T^{\prime \prime}\left(i d_{L_{\infty}(\mu)}-A\right)\left(f_{i}\right), x_{i}^{\prime}\right\rangle\right|+\left|\sum_{i=1}^{n}\left\langle T^{\prime \prime}\left(A\left(f_{i}\right)\right), x_{i}^{\prime}\right\rangle\right| \\
& \leq \varepsilon\|T\| \sum_{i=1}^{n}\left\|f_{i}\right\|\left\|x_{i}^{\prime}\right\|+\left|\sum_{i=1}^{n}\left\langle T^{\prime \prime}\left(A\left(f_{i}\right)\right), x_{i}^{\prime}\right\rangle\right| .
\end{aligned}
$$

As $T$ is a lattice homomorphism, according to Ando (see [8: Theorem 1.4.19]) $T^{\prime \prime}$ is also a lattice homomorphism, and $X_{1}=T^{\prime \prime}\left(M_{1}\right)$ is a finite-dimensional sublattice of $X^{\prime \prime}$. From the theorem of Conroy and Moore (see [2: Lemma 4.3]), $X^{\prime \prime}$ is lattice finitely representable in $X$, and then there is a finite-dimensional sublattice $X_{2}$ of $X$ and a lattice isomorphism $C: X_{1} \rightarrow X_{2}$ such that $\|C\|\left\|C^{-1}\right\| \leq 1+\varepsilon$. As $X$ is lattice finitely representable in $\ell_{1}\left\{\ell_{H}\right\}$, there is a finite-dimensional sublattice $Z$ of $\ell_{1}\left\{\ell_{H}\right\}$ and an lattice isomorphism $D: X_{2} \rightarrow Z$ such that $\|D\|\left\|D^{-1}\right\| \leq 1+\varepsilon$. Let $R: M_{1} \rightarrow Z$ denote the map $R=D C T^{\prime \prime}$ and $I_{Z}$ the inclusion of $Z$ in $\ell_{1}\left\{\ell_{H}\right\}$. Then

$$
\begin{aligned}
\sum_{i=1}^{n}\left\langle T^{\prime \prime}\left(A\left(f_{i}\right)\right), x_{i}^{\prime}\right\rangle & =\sum_{i=1}^{n}\left\langle\left((D C)^{-1}(D C) T^{\prime \prime}\right)\left(A\left(f_{i}\right)\right), x_{i}^{\prime}\right\rangle \\
& =\sum_{i=1}^{n}\left\langle\left((D C)^{-1}\right)\left(R\left(A\left(f_{i}\right)\right)\right), x_{i}^{\prime}\right\rangle \\
& =\sum_{i=1}^{n}\left\langle R\left(A\left(f_{i}\right)\right),\left((D C)^{-1}\right)^{\prime}\left(x_{i}^{\prime}\right)\right\rangle \\
& =\left\langle R, \sum_{i=1}^{n} A\left(f_{i}\right) \otimes\left((D C)^{-1}\right)^{\prime}\left(x_{i}^{\prime}\right)\right\rangle
\end{aligned}
$$

with

$$
\sum_{i=1}^{n} A\left(f_{i}\right) \otimes\left((D C)^{-1}\right)^{\prime}\left(x_{i}^{\prime}\right) \in M_{1} \otimes Z^{\prime} .
$$

The map $I_{Z}^{\prime}:\left(\ell_{1}\left\{\ell_{H}\right\}\right)^{\prime} \rightarrow Z^{\prime}$ is a canonical quotient map and $M_{1}$ is a $\mathcal{L}_{\infty, 1+\varepsilon}$ space. Then after Proposition 10, there is $u \in M_{1} \otimes\left(\ell_{1}\left\{\ell_{H}\right\}\right)^{\prime}$ with a representation $u=\sum_{j=1}^{m} g_{j} \otimes a_{j}$ so that

$$
\begin{gathered}
\left(i d_{M_{1}} \otimes I_{Z}^{\prime}\right)(u)=\sum_{i=1}^{n} A\left(f_{i}\right) \otimes\left((D C)^{-1}\right)^{\prime}\left(x_{i}^{\prime}\right) \\
\left(g_{H}^{c}\right)^{\prime}\left(u ; M_{1} \otimes\left(\ell_{1}\left\{\ell_{H}\right\}\right)^{\prime}\right) \leq(1+\varepsilon)\left(g_{H}^{c}\right)^{\prime}\left(\sum_{i=1}^{n} A\left(f_{i}\right) \otimes\left((D C)^{-1}\right)^{\prime}\left(x_{i}^{\prime}\right) ; M_{1} \otimes Z^{\prime}\right) .
\end{gathered}
$$


Then

$$
\begin{aligned}
\left\langle R, \sum_{i=1}^{n} A\left(f_{i}\right) \otimes\left((D C)^{-1}\right)^{\prime}\left(x_{i}^{\prime}\right)\right\rangle & =\left\langle R,\left(i d_{M_{1}} \otimes I_{Z}^{\prime}\right)(u)\right\rangle \\
& =\sum_{j=1}^{m}\left\langle R\left(g_{j}\right), I_{Z}^{\prime}\left(a_{j}\right)\right\rangle \\
& =\sum_{j=1}^{m}\left\langle\left(I_{Z} R\right)\left(g_{j}\right), a_{j}\right\rangle \\
& =\left\langle I_{Z} R, u\right\rangle .
\end{aligned}
$$

But $I_{Z} R: M_{1} \rightarrow \ell_{1}\left\{\ell_{H}\right\}$ is a positive map. Then from Theorem $4, I_{Z} R$ is $H^{c}$-integral. Accordingly,

$$
\begin{aligned}
\left|\left\langle I_{Z} R, u\right\rangle\right| & \leq\left\|I_{Z} R\right\|\left(g_{H}^{c}\right)^{\prime}\left(u ; M_{1} \otimes\left(\ell_{1}\left\{\ell_{H}\right\}\right)^{\prime}\right) \\
& \leq(1+\varepsilon)\|R\|\left(g_{H}^{c}\right)^{\prime}\left(\sum_{i=1}^{n} A\left(f_{i}\right) \otimes\left((D C)^{-1}\right)^{\prime}\left(x_{i}^{\prime}\right) ; M_{1} \otimes Z^{\prime}\right) \\
& \leq(1+\varepsilon)\|D\|\|C\|\left\|T^{\prime \prime}\right\|\left\|(D C)^{-1}\right\|\left(g_{H}^{c}\right)^{\prime}\left(\sum_{i=1}^{n} A\left(f_{i}\right) \otimes x_{i}^{\prime} ; M_{1} \otimes N\right) \\
& \leq(1+\varepsilon)^{3}\|T\|\|A\|\left(g_{H}^{c}\right)^{\prime}(z ; M \otimes N) \\
& \leq(1+\varepsilon)^{4}\|T\|\left(g_{H}^{c}\right)^{\prime}(z ; M \otimes N) \\
& \leq(1+\varepsilon)^{4}\|T\|\left(\left(g_{H}^{c}\right)^{\prime}\left(z ; L_{\infty}(\mu) \otimes X^{\prime}\right)+\varepsilon\right) .
\end{aligned}
$$

In consequence,

$$
\left|\left\langle T^{\prime \prime}, z\right\rangle\right| \leq \varepsilon\|T\| \sum_{i=1}^{n}\left\|f_{i}\right\|\left\|x_{i}^{\prime}\right\|+(1+\varepsilon)^{4}\|T\|\left(\left(g_{H}^{c}\right)^{\prime}\left(z ; L_{\infty}(\mu) \otimes X^{\prime}\right)+\varepsilon\right) .
$$

Then as $\varepsilon$ is arbitrary,

$$
\left|\left\langle T^{\prime \prime}, z\right\rangle\right| \leq\|T\|\left(g_{H}^{c}\right)^{\prime}\left(z ; L_{\infty}(\mu) \otimes X^{\prime}\right)
$$

hence

$$
T^{\prime \prime} \in\left(L_{\infty}(\mu) \otimes_{\left(g_{H}^{c}\right)^{\prime}} X^{\prime}\right)^{\prime} \quad \text { with }\left\|T^{\prime \prime}\right\|_{\left(L_{\infty}(\mu) \otimes_{\left(g_{H}^{c}\right)^{\prime}} X^{\prime}\right)^{\prime}} \leq\|T\|=\left\|T^{\prime \prime}\right\| .
$$

Then $T^{\prime \prime}$ is $H^{c}$-integral, hence $T$ is also $H^{c}$-integral with $H^{c}$-integral's norm being less or equal to $\|T\|$

Let $D$ be an index set and $\mathcal{D}$ a non-trivial ultrafilter on $D$. Given a family of Banach spaces $\left\{A^{d}\right\}_{d \in D},\left(A_{d}\right)_{\mathcal{D}}$ denotes the corresponding ultraproduct. If every $A^{d}$ is a Banach lattice, $\left(A^{d}\right)_{\mathcal{D}}$ has a canonical order which makes it a Banach lattice. If we have another family of Banach spaces $\left\{B_{d}\right\}_{d \in D}$ and a family of operators $\left\{T^{d} \in \mathcal{L}\left(A^{d}, B^{d}\right)\right\}_{d \in D}$ such that $\sup _{d \in D}\left\|T^{d}\right\|<\infty,\left(T^{d}\right)_{\mathcal{D}} \in \mathcal{L}\left(\left(A^{d}\right)_{\mathcal{D}},\left(B^{d}\right)_{\mathcal{D}}\right)$ denotes the canonical ultraproduct 
operator. The main ideas on ultraproducts of Banach spaces used in this paper are stated in $[2,3]$. The importance of the ultraproduct construction techniques in operators theory is well known. Therefore the interest of finite representability in this paper goes through the fundamental fact that a Banach space (lattice) $X$ is finitely representable (lattice finitely representable) in the family of Banach spaces (lattices) $\left\{X_{i}\right\}_{i \in I}$ (in the Banach space (lattice) $Y$ ), if and only if $X$ is isometric to a subspace (sublattice) of some ultraproduct (ultrapower) of Banach spaces (lattices) of that family (of $Y$ ). Moreover, according to Conroy and Moore's theorem, the bidual $E^{\prime \prime}$ of a Banach lattice $E$ is lattice finitely representable in $E$ (see [2: Lemma 4.3]). Finally, we have the following characterization theorem of $H^{c}$-integral operators:

Theorem 11. For every pair of Banach spaces $E$ and $F$, the following statements are equivalent:

1) $T \in \mathcal{I}_{H^{c}}(E, F)$.

2) $J_{F} T$ factors in the following way:

where $B$ is a lattice homomorphism and $X$ a Banch space, which is lattice finitely representable in $\ell_{1}\left\{\ell_{H}\right\}$. Furthermore, $\mathbf{I}_{H^{c}}(T)=\inf \{\|D\|\|B\|\|A\|\}$, taking the infimum over all such factors.

Proof. The implication 2) $\Rightarrow 1$ ) is evident from the preceding theorem. As to 1) $\Rightarrow 2$ ), we define the set

$$
D=\left\{(M, N): M \in F I N(E) \text { and } N \in F I N\left(F^{\prime}\right)\right\}
$$

where $\operatorname{FIN}(Y)$ is the set of finite-dimensional subspaces of a Banach space $Y$, endowed with the natural inclusion order

$$
\left(M_{1}, N_{1}\right) \leq\left(M_{2}, N_{2}\right) \quad \Longleftrightarrow \quad M_{1} \subset M_{2} \text { and } N_{1} \subset N_{2} .
$$

For every $\left(M_{0}, N_{0}\right) \in D$, set

$$
\begin{aligned}
R\left(M_{0}, N_{0}\right) & =\left\{(M, N) \in D:\left(M_{0}, N_{0}\right) \subset(M, N)\right\} \\
\mathcal{R} & =\{R(M, N):(M, N) \in D\} .
\end{aligned}
$$

$\mathcal{R}$ is a filter basis in $D$, and according to Zorn's lemma, let $\mathcal{D}$ be an ultrafilter on $D$ containing $\mathcal{R}$. If $d \in D, M_{d}$ and $N_{d}$ denote the finite-dimensional subspaces of $E$ and $F^{\prime}$, respectively, so that $d=\left(M_{d}, N_{d}\right)$. For every $d \in D$, if $z \in M_{d} \otimes N_{d}$,

$$
J_{F} T_{\mid M_{d} \otimes N_{d}} \in\left(M_{d} \otimes_{\left(g_{H}^{c}\right)^{\prime}} N_{d}\right)^{\prime}=M_{d}^{\prime} \otimes_{g_{H}^{c}} N_{d}^{\prime}=\mathcal{N}_{H^{c}}\left(M_{d}, N_{d}^{\prime}\right) .
$$


Then from the characterization of $H^{c}$-nuclear operators, Theorem $3, J_{F} T_{\mid M_{d} \otimes N_{d}}$ factors in the following way:

where $B^{d}$ is a positive diagonal with $I_{H^{c}}\left(T_{\mid M_{d} \otimes N_{d}}\right) \leq I_{H^{c}}(T)$. Without loss of generality we can assume that $\left\|A^{d}\right\|=\left\|C^{d}\right\|=1$ and $\left\|C^{d}\right\| \leq I_{H^{c}}(T)$. We define $W_{E}: E \rightarrow\left(M_{d}\right)_{\mathcal{D}}$ by $W_{E}(x)=\left(x^{d}\right)_{\mathcal{D}}$, so that $x^{d}=x$ if $x \in M_{d}$ and $x^{d}=0$ if $x \notin M_{d}$. In the same way we define $W_{F^{\prime}}: F^{\prime} \rightarrow\left(N_{d}\right)_{\mathcal{D}}$ by $W_{F^{\prime}}(a)=\left(a^{d}\right)_{\mathcal{D}}$, so that $a^{d}=a$ if $a \in N_{d}$ and $a^{d}=0$ if $a \notin N_{d}$. Then we have the following commutative diagram:

Then with $A=\left(A^{d}\right) \mathcal{D}, B=\left(B^{d}\right) \mathcal{D}, C=\left(C^{d}\right) \mathcal{D}$, and $G=\left(\ell_{\infty}\left\{\ell_{\infty}\left(\Gamma^{d}\right)\right\}\right)_{\mathcal{D}}$ and $Z=\left(N_{d}^{\prime}\right)_{\mathcal{D}}$, we have the following diagram:

As $G$ is an abstract $M$-space, $G^{\prime \prime}$ is lattice isometric to some $L_{\infty}(\mu)$. Moreover, $F^{\prime \prime}$ is complemented in $F^{\prime \prime \prime \prime}$, and if $P: F^{\prime \prime \prime \prime} \rightarrow F^{\prime \prime}$ is the projection, then $\|P\| \leq 1$ and $P J_{F^{\prime \prime}} J_{F} T=J_{F} T$. According to this we have the following diagram:

But $Z$ is lattice finitely representable in $\ell_{1}\left\{\ell_{H}\right\}$, and according to the theorem of Conroy and Moore, $Z^{\prime \prime}$ is also lattice finitely representable in $\ell_{1}\left\{\ell_{H}\right\}$ 


\section{References}

[1] Defant, A. and K. Floret: Tensor Norms and Operator Ideals (North Holland Math. Studies). Amsterdam: North Holland Publ. Comp. 1993.

[2] Haydom, R., Levy, M. and Y. Raynaud: Randomly Normed Spaces. Paris: Hermann 1991.

[3] Heinrich, S.: Ultraproducts in Banach spaces theory. J. Reine Angew. Math. 313 (1980), $72-104$.

[4] Hollstein, R.: Inductive limits and $\varepsilon$-tensor products. J. Reine Angew. Math. 319 (1980), $38-62$.

[5] Lindenstrauss, J. and L. Tzafriri: Classical Banach Spaces, Vol. II. Berlin et al.: SpringerVerlag 1979.

[6] Loaiza, G., López Molina, J. A. and M. J. Rivera: Normas tensoriales y espacios de operadores factorables a través de espacios de Orlicz.. Revista Mat. de la Univ. Tecnológica de Pereira (to appear).

[7] Loaiza, G., López Molina, J. A. and M. J. Rivera: On absolutely summing, nuclear and integral operators associated to an Orlicz function. Preprint.

[8] Meyer-Nieberg, P. Banach Lattices. Berlin et al.: Springer-Verlag 1991.

[9] Schaefer, H. H.: Banach Lattices and Positive Operators. Berlin et al.: Springer-Verlag 1974.

Received 01.10.2000; in revised form 02.02.2001 